\title{
An Experiment on a Core Controversy*
}

\author{
Huibin Yan $^{\dagger} \quad$ Daniel Friedman ${ }^{\ddagger} \quad$ David Munro ${ }^{\S}$
}

February 15, 2016

\begin{abstract}
A longstanding criticism of the core is that it is too sensitive to small changes in player numbers, as in a well known example where one extra seller (resp. buyer) causes the entire surplus to go to the buyer's (seller's) side. We test this example in the lab, using several different trading institutions. We find that successful collusion is relatively infrequent and decreasing over time even with institutions that facilitate collusion and, consistent with core theory, a disproportionate share of the surplus typically goes to the less numerous side. Our study also illuminates the boundaries of competitive equilibrium.
\end{abstract}

Keywords: core, collusion, experiment, fairness, market

JEL Codes: C71, C78, C92, D43

${ }^{*}$ We are grateful to Timothy Cason, Yan Chen, Rachel Croson, Steve Gjerstad, Chenying Huang and Ryan Oprea for helpful comments, to Todd Swarthout for help with MarketLink software, and to the National Science Foundation (grant IIS-0527770) and the UCSC Social Sciences Division for financial support. We also thank Tom Campbell, Olga Rud, Kuntal Kumar Das, Tomera Ten, Nan Geng and especially Yuan Yuan for patient research assistance. Finally, we are indebted to a very patient editor of this journal and to two anonymous referees for thoughtful suggestions on sharpening the presention.

${ }^{\dagger}$ LEEPS Lab, University of California Santa Cruz hbinyan@gmail.com

${ }^{\ddagger}$ Department of Economics, University of California Santa Cruz dan@ucsc.edu

${ }^{\S}$ Department of Economics, University of California Santa Cruz dmunro@ucsc.edu 


\section{Introduction}

The concept of the core is generally regarded as the most important contribution to economics made by cooperative game theory. Among other things, it provides a game theoretic foundation for the competitive equilibrium (CE). A well known critique of the core, however, is its seemingly excessive sensitivity to small changes in the numbers of players. The standard example is a market game in which each buyer and each seller wants to transact a single indivisible unit of a good. All buyers have the same value $v$ and all sellers have the same cost $c<v$. The core predicts a unique outcome when there are unequal numbers of buyers and sellers, namely that all the trade surplus goes to the less numerous side (called the short side hereafter) of the market. In particular, one extra trader on one side shifts the entire surplus to the other side.

The critique holds that this extreme sensitivity is unrealistic. For example, the long side traders (the more numerous group, slated to receive zero payoff) may find a way to collude and seize some of the surplus. Or, as suggested by recent work in behavioral economics, fairness norms might cause traders on either side to reject these extreme allocations. If both forces work in tandem, we might even see fairness-motivated collusion.

In this paper we take the critique to the laboratory. To give collusion its best shot, we examine small number cases, with two or three players on the long side and exactly one fewer player on the short side. Core theory does not specify the trading institution, so we look at several. At one extreme, we use standard computerized continuous double auction market (DA-Std henceforth). We also consider two augmentations that provide collusion opportunities. One augmentation (DA-Chat) allows free pretrade communication in computer chatrooms. Another augmentation (DA-Barg) keeps the chatroom open throughout the trading period and also facilitates and implements collusive profit sharing agreements. Finally, at the other extreme, we drop market-like features and consider a direct negotiation chatroom (Barg) that allows traders to implement enforceable trade agreements.

Section 2 reviews relevant literature. The most closely related laboratory studies are some pioneering market experiments conducted in the 70's, and there are also related experiments in political science and on market competitivity. Section 3 reviews both the cooperative and the noncooperative theories of the core, and notes their implications for our laboratory study. 
Section 4 lays out our experimental design and the testable implications. Section 5 presents the results, and Section 6 summarizes and discusses implications. On-line Appendix A presents supplementary regressions and robustness checks, Appendix B details the coding of chatroom dialogues and collusion activity, and Appendix $\mathrm{C}$ reproduces sample instructions for subjects.

\section{Related Literature}

The core was introduced formally in Shapley (1952) and Gillies (1954), though its conceptual roots go back to Edgeworth (1881). A more recent theoretical literature following Selten (1980) builds noncooperative foundations for the core through constructing noncooperative bargaining games from which core and only core outcomes emerge in equilibrium; see Perry and Reny (1994) for a prominent example, and see the introduction in Yan (2003) for a brief survey. Insights from this recent literature inform our implementation, as noted in section 3.2 below.

In a pioneering series of experiments, Murnighan and Roth (1977, 1978, 1980) tested core prediction for the extreme example (framed as "left-shoe, right-shoe") against other cooperative solution concepts. Using just one short sider and two to eleven long siders and using bargaining institutions far different from ours, ${ }^{1}$ they found that better information and communication opportunities fostered more effective collusion among long siders. With two long siders, their short sider on average kept less than $60 \%$ of the total surplus in most treatments, and even in their minimal information and communication treatments the short sider seldom averaged more than $70 \%$ of the surplus.

About the same time, Fiorina and Plott (1978) launched a laboratory investigation of the core (and other cooperative solution concepts) in voting games. They found the core did a good job of predicting the outcome of majority rule voting, although Eavey and Miller (1984) found that "fair" alternatives outside the core did even better.

Because the core generally contains the competitive equilibrium (CE), the unique core allocation in our example coincides with CE. The next section will discuss theoretical connec-

\footnotetext{
${ }^{1}$ They used fixed matchings, unpaid subjects or subjects participating for course credit, and sequences of simultaneous bilateral offers - e.g., one long sider might offer to buy the other long sider's shoe first and if successful then offer to buy the short sider's shoe.
} 
tions, but here it is worth mentioning the empirical literature known as boundary studies of CE. Smith and Williams (1990) report early computerized market studies, including several sessions with identical valuations and identical costs. These sessions featured a continuous double auction market (CDA) with 4 multi-unit buyers and 4 multi-unit sellers and sizable imbalances (either 16 units demanded and 11 units supplied, or the reverse). They found convergence to near the CE, with over $90 \%$ of surplus typically going to the short side after 3 or 4 periods. Also inducing identical valuations and identical costs, Friedman and Ostroy (1995) run 4-buyer-4-seller (4x4 hereafter) standard CDA markets with slightly imbalanced demand and supply parameters but with essentially divisible goods, and they found slow convergence towards the CE price. Isaac et al. (1984) allowed one side of a $4 \times 4$ market to collude, in the form of a one-time face-to-face free communication for up to 4 minutes when the session was paused, and still found rather competitive outcomes in the CDA. Joyce (1984) studied collusion in a hybrid market institution with some CDA and some Call Market features and reported that buyers were better than sellers at colluding and extracting surplus.

Our experiment differs from those studies in having only 1-3 traders on each side of the market, facilitating collusion. Also, we publicly announce the demand and supply parameters as suggested by core theory, while CE studies typically treats them as private information, so subjects initially may not be aware of their market power. ${ }^{2}$

\section{Theoretical Background}

Intuitively, the core consists of allocations that are stable in the sense that no subgroup of players can break away and achieve better payoffs for all its members.

To formalize, let $N$ denote the set of all players. Any nonempty subset $T \subseteq N$ of players is called a coalition, and $|T| \geq 1$ denotes the number of players in $T$. The grand coalition

\footnotetext{
${ }^{2}$ Readers may be reminded of other laboratory experiments that, on closer scrutiny, are less closely related to our experiment. As a counterpoint of their finding of cross-country differences in ultimatum game outcomes, Roth et al. (1991) used 1-seller-versus-9-buyer market games for control, showing that in all the countries the market games lead to the same CE outcome in which all surplus goes to the seller. In their setting, no individual buyer has any impact on the equilibrium price, a crucial difference from our experiment. Like us, Cason and Noussair (2007) studied very thin markets, with only 2 single unit buyers and 3 single unit sellers. But they investigated price dispersion when there were search frictions in Posted Offer markets, an issue almost polar to ours.
} 
consists of all players, $T=N$. A characteristic function is a mapping $v$ from the set of all coalitions into $\Re_{+}$. The number $v(T)$ is called the worth of coalition $T$. The pair $(v, N)$ defines cooperative game with transferable utility.

An allocation for coalition $T$ is an element in $\Re_{+}^{|T|}$, written as $\omega=\left(\omega_{i}\right)_{i \in T}$. It is feasible if $\sum_{i \in T} \omega_{i} \leq v(T)$. A coalition $T$ is said to block an allocation for the grand coalition $\omega=\left(\omega_{i}\right)_{i \in T}$ if $v(T)>\sum_{i \in T} \omega_{i}$. The core is the set of all the feasible allocations for the grand coalition that cannot be blocked by any coalition.

\subsection{The Buyer-Seller Example as a Cooperative Game}

Now let $N$ be the union of two disjoint player subsets, $B$ and $S$, called buyers and sellers respectively. Let the worth of any coalition of one buyer and one seller be 1, interpreted as the total trade surplus in one transaction. Then the set of worth of any coalition $T$ equal to the number of buyer-seller pairs in $T$, that is, $v(T)=\min \{|B \cap T|,|S \cap T|\}$. The game is called a buyers' market, and $B$ is called the short side, if $|B|<|S|$. The opposite case, $|B|>|S|$, is called a sellers' market because now $S$ is the short side. The long side, of course, is the other subset of players: $S$ in a buyers' market and B in a sellers' market. ${ }^{3}$

The core in such games is unique, non-empty, and extreme: the entire surplus goes to the short side players, who divide it equally. While this result is well known, we find no published proof, so we provide one here.

Proposition 1. A buyers' (sellers') market has a unique core allocation $\omega$. We have $\omega_{i}=1$ if player $i$ is a short sider and otherwise $\omega_{i}=0$.

Proof. Consider a buyers' market. We will first show that, fixing any seller $i$, he must get a zero payoff in any core allocation $\omega$. Consider the coalition of the remaining players $T=N \backslash\{i\}$. By the definition of buyers' market, T still contains at least as many sellers as buyers. Hence the specification of $v$ ensures that $T$ has the same worth as the grand coalition, i.e. $v(T)=v(N)$. Hence $T$ blocks any allocation that gives a positive payoff to $i$. Therefore, any core allocation $\omega$ must satisfy $\omega_{i}=0$.

\footnotetext{
${ }^{3}$ The same characteristic function is sometimes interpreted in terms of the sets $B=L$ and $S=R$ of owners of left shoes and right shoes, or other Leontief-complementary pairs of goods.
} 
We now show that each buyer gets 1 . Remember that any coalition consisting of one buyer and one seller has worth 1 , so for any $i \in S$ and $j \in B$, we must have $\omega_{i}+\omega_{j} \geq 1$. Since $\omega_{i}=0$, we must have $\omega_{j} \geq 1$. But with each buyer getting at least 1 , the only feasible allocation is that each buyer gets exactly $\omega_{j}=1$.

It is easy to see that no subcoalition blocks $\omega$ : for any $T \subseteq N$, we have $\sum_{i \in T} \omega_{i}=|B \cap T| \geq$ $\min \{|B \cap T|,|S \cap T|\}=v(T)$. So the core allocation is indeed unique and as specified. The sellers' market is symmetric.

The intuition is transparent in a market with one buyer and two sellers. Any proposed allocation that gives either seller a positive payoff leaves a joint payoff of less than one to the buyer and the other seller, who can form a coalition that blocks the proposed allocation.

In a typical market transaction, no transfer (i.e. profit sharing) is possible between traders on the same side, so a trader unable to trade must receive zero. This can be captured by restricting feasible allocations to those assigning zero payoffs to redundant traders. In a market with one buyer and two sellers for example, at least one seller receives zero in any allocation in this restricted (partially transferable utility) game. It is easy to see that the above intuition, and indeed the proof of Proposition 1 and hence the unique core prediction, holds for the restricted game as well.

Recall the critique that collusion among long siders may cause the core prediction to fail. Here "collusion" should not be interpreted as forming a coalition, since long siders by themselves can only form zero-worth coalitions. Rather, to make sense of the critique, we should interpret collusion as refusing to join a coalition that would block an allocation helpful to a same sider, i.e., in market terminology, not undercutting that same sider's price. This approach extends to the unrestricted game where transfers are possible among same siders. Consider an allocation in which a long sider receives a transfer (a positive payoff) for not participating in trade. The other traders could block that allocation because they could divide that transfer among themselves. Here collusion takes the form of agreeing not to block the transfer. ${ }^{4}$

\footnotetext{
${ }^{4}$ How large might that transfer be? It seems to us unlikely that long siders could maintain a collusion that promised anyone more than the per capita surplus, since egalitarian sentiments (easily voiced in a chatroom) plus self interest would encourage other long siders to undercut that promise and thus block the proposed allocation.
} 
As mentioned in the Introduction, the unique core allocation coincides with the $\mathrm{CE}$ in our example. To see this, consider a market where all sellers have the same opportunity cost $c \geq 0$ and all buyers have the same reservation value $r=c+1$. Then the surplus (potential gains from trade) within any coalition is as specified in the worth function above. The CE is by definition a price $p$ and post-trade allocation such that desired sales at $p$ equal desired purchases. It is easy to see that the unique CE price is $p=r$ in a sellers' market and is $p=c$ in a buyers' market. For example, the flat demand and supply configuration in Figure 1 arise in a 2x3 game where sellers have unit cost LD55 and buyers have unit value LD89. At CE, the total surplus is 2 units (a unit here being LD89 - 55 = LD34) and goes entirely to the short side, the buyers.

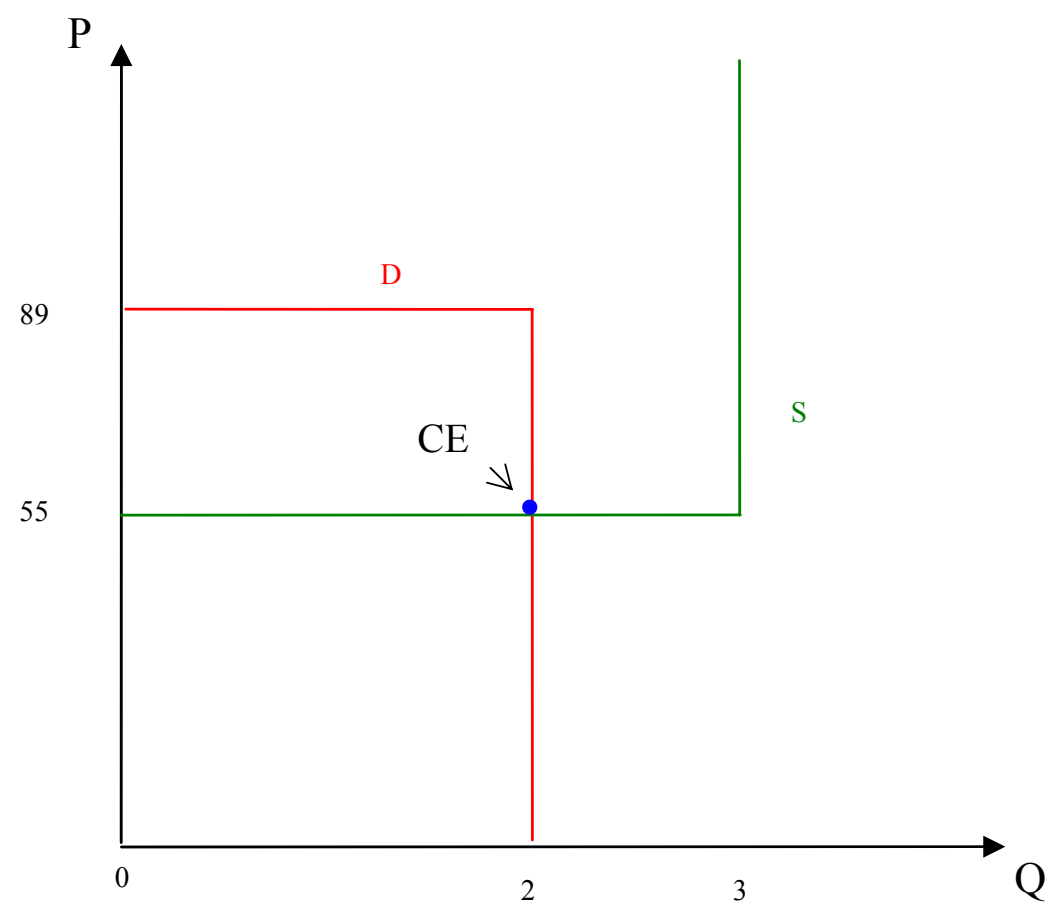

Figure 1: Demand, supply and competitive equilibrium (CE) in a 2x3 buyer's market

So is our study a test of the core or of CE? The underlying premise of CE is that all traders are price takers, but in the present example even long siders have potential market power in that they can each dramatically affect the set of market clearing prices. Also, with the small number of traders and the collusion opportunities we provide, CE theory is much more equivocal that than is the core. Moreover, full implementation of core theory requires 
that transfers among same siders be allowed, and such agreements are infeasible in the usual CE testing environment. Therefore we regard our study primarily as a test of the core, and secondarily as a stress test of CE.

\subsection{Implementation Issues}

As can be seen from the discussion above, the (cooperative) theory of the core places few constraints on how players interact. In choosing a laboratory implementation, we seek guidance from recent advances in both theoretical and experimental economics. The implementation should give collusion a good chance while remaining compatible with cooperative and noncooperative theory.

The literature on the noncooperative foundations of the core seeks to construct noncooperative bargaining games that generate core and only core outcomes in equilibrium. Shared features of these games include complete and perfect information, and one-shot interactions. Our implementation also shares those features.

To see the need for complete and perfect information, recall the blocking coalition described right after the proof of the proposition. That buyer and the other seller must be aware that they can form the coalition and split its worth of 1 in a way that leaves both better off than in the proposed allocation. To ensure such awareness, all buyer values and all seller costs should be public. Indeed, as part of perfect information, players need to know the offers being made to a coalition in order to see whether it blocks another coalition. Conversely, the core outcome (with full surplus extraction by one side of the market) would never arise in equilibrium with private values and costs, as is well known in the mechanism design literature. ${ }^{5}$

It is well known in noncooperative game theory that access to commitment devices, and to binding agreements in particular, often drastically changes the outcome of a game. The noncooperative foundations literature typically allows binding agreements only for outcomes prescribed by the characteristic (i.e., worth) function. This restriction rules out, in particular, binding agreement between long siders where one side sells his trading right. It is not hard to

\footnotetext{
${ }^{5}$ The intuition is that with values and costs believed to be randomly drawn from some probability distributions, a long sider, say a buyer, (rationally) believes that he can always (except when his is the lowest possible value) bid a bit below his true value and still have positive probability of transacting and hence earn positive expected payoff, assuming that the other buyers bid their true values.
} 
show that the core prediction would not be an equilibrium outcome if such agreements were allowed.

Finally, the core pertains to one-shot interactions - a coalition blocks only if it can split its current worth to mutual immediate benefit. All sorts of outcomes are possible in repeated interaction, in which players sacrifice now in the hope of getting greater future gains. Such arrangements, however, are outside the scope of core theory. Thus, in testing the core prediction, we shall avoid repeatedly matching the same individual buyers and sellers.

Although guided by those shared features, we do not directly implement a model from the noncooperative foundations literature, for two reasons. First, that literature seeks to support only core outcomes in equilibrium, and our results will be much stronger if the core outcome emerges from a procedure that allows noncore as well as core outcomes in equilibrium. The second reason is behavioral. Bargaining in those models typically proceeds one proposal at a time, and each proposal specifies a feasible allocation for some coalition whose members immediately vote on it. It is implemented if all members vote in favor, but if even one member opposes it then it is discarded and someone can make a new proposal. In our context this means that the short sider can veto any long sider proposal involving the short sider, and since any feasible allocation involving positive payoffs to long siders necessarily involves at least one short sider, the short sider could immediately take off the table any long side proposal attempting collusion. This would seem not to give collusion a fair shot.

For both reasons we begin with a natural market trading institution, DA-Std, in which a trader's bid or offer remains valid until retracted. Decades of market experiments show that this institution, the continuous double auction, reliably produces convergence in brief trading periods in the lab, and it is used widely in practice (e.g. in the Chicago trading pits, the NYSE, and new electronic exchanges). Furthermore, it is neutral, in the sense that a priori it does not favor either side of the market.

Beyond this, we seek institutions that enable unstructured bargaining and collusion. As detailed in the next section, our institution DA-Chat augments the standard continuous double auction with free pre-play communication in a chatroom, and the new institution DA-Barg further enables negotiation and enforcement of collusive profit sharing agreements. Last, in an attempt to push the core prediction to its limits, we test another new institution, Barg, that 
drops the market aspects while continuing to enable and enforce bilateral and multilateral collusive agreements. The core prediction applies in all four institutions even though collusion opportunities differ.

How many buyers and sellers should we use? To challenge the core prediction, we will use just one extra long side trader, that is, $n \times(n+1)$ market games. While the core prediction may seem more striking for larger numbers of players, it is well known that collusion is harder to sustain when more people are involved (e.g., Dufwenberg and Gneezy (2000), Huck et al. (2004), Isaac et al. (1984), and Harrison and McKee (1985). $)^{6}$ Therefore we will use the minimal numbers of traders, with $n=1,2$ as the number of short siders.

\section{The Experiment}

\subsection{The Trading Institutions}

In DA-Std, the standard continuous double auction, sellers and buyers can submit bids and asks at any time, simply by dragging a slider or typing in the desired price, and clicking a Bid or Ask button. These offers are displayed on the screens of every trader in the marketplace, as in Figure 2. Transactions occur immediately at the older price whenever a bid and ask overlap, or when a trader selects an offer from the other side of the market and clicks the Buy or Sell button. DA-Std trading lasts 60 (for 1x2 sessions) or 95 (for 2x3 sessions) seconds each period.

DA-Chat sessions begin with two standard CDA periods (excluded from main data analysis), as it rarely occurred to our pilot subjects to use the chat facility for collusion in the first periods. ${ }^{7}$ Period three and subsequent trading periods are preceded by 2 (for $1 \mathrm{x} 2$ markets) or 3 (for 2x3 markets) minutes' pre-play communication. Subjects enter a computerized chatroom where they may type messages seen by everyone in their marketplace. Such talk is cheap in that there is no enforcement mechanism. The bids and asks in the CDA are shown without revealing the trader's identity, e.g. seller A or B, in order to prevent the short sider(s)

\footnotetext{
${ }^{6}$ Our own results confirm that indeed collusion is harder in $2 \mathrm{x} 3$ than in $1 \mathrm{x} 2$ markets; see, e.g., Table 2.

${ }^{7}$ We found no evidence of anchoring effects from having standard CDA in the first two periods; see Appendix A.1. Skipping the chatroom in these periods saves about 10 minutes in total and keeps the session within 2 hours.
} 


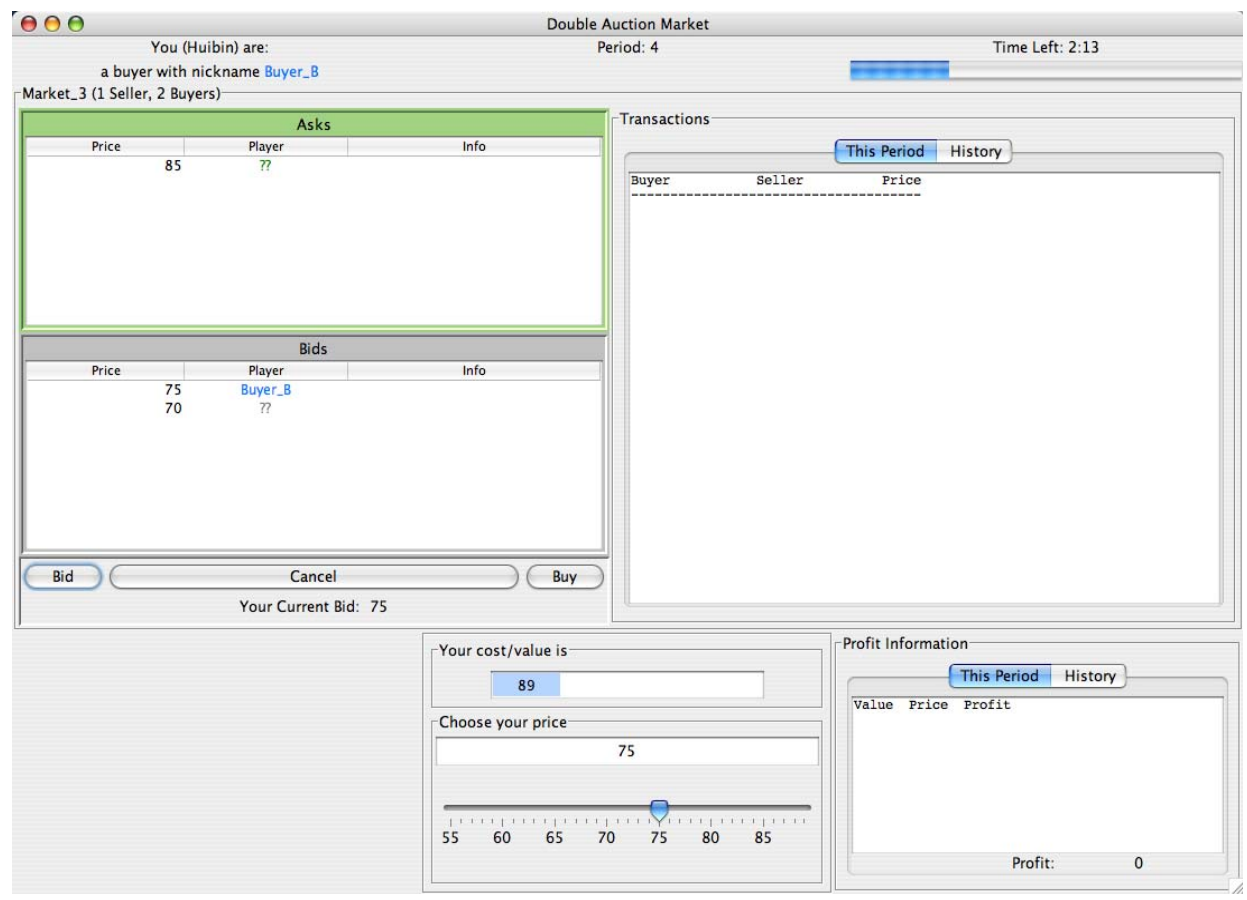

Figure 2: DA-Std User Interface: DA-Std trading screen is shown for a buyer (ID code Buyer$\mathrm{B}$, actual name Huibin). She can post a bid by either typing the desired price or by dragging the slider to the desired value in the "Choose your price" box and then clicking the Bid button. The value of 89 is shown nearby against a blue shaded background. The large box at upper left, labeled "Market 3 (1 Seller, 2 Buyers)," shows all current bids by both buyers and asks by the seller. The buyer transacts either by clicking on an ask (or bidding higher than some ask) or by waiting for the seller to click on (or cross) her bid. The "Transactions" box shows the current period transactions in her local market; by clicking on the History tab the previous period's transactions are shown. The "Profit Information" box at the bottom right of the screen shows Buyer-B's previous transactions and profit. The History tab details trading profits and transactions in earlier periods. Sellers' trading screens are similar, with Ask and Sell buttons in the center box, costs instead of values, etc.

from punishing the long sider who initiates collusion in the chatroom.

Unlike DA-Std and DA-Chat, the third institution, DA-Barg, permits transfers among long siders. Adapting a MarketLink feature, DA-Barg opens a parallel collusion market, referred to as "Market 2" on traders' screens, in addition to leaving the chatroom open throughout each 3-minute trading period. In Market 2, as shown in Figure 3, long side traders can negotiate contracts to collude. They click tabs to switch between this screen and "Market 1", the standard MarketLink CDA supplemented with a bulletin board that reports all activities in both markets. In a 1x2 sellers' market, for example, the two buyers negotiate in Market 2 to have one buyer give up the right to trade in Market 1 in return for some percentage of the 


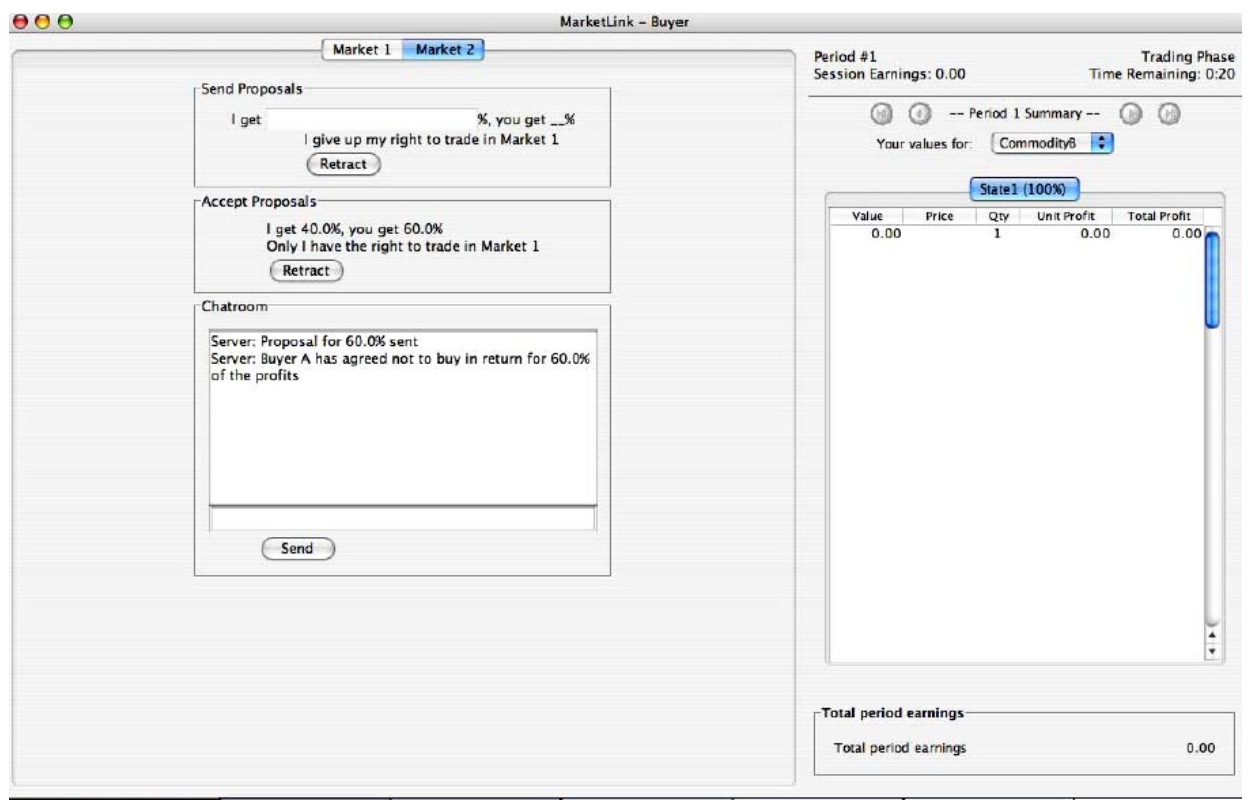

Figure 3: DA-Barg User Interface: DA-Barg trading screen, tabbed to Market 2, is shown for Buyer-B. The box at upper left allows the buyer to propose (and to retract) an offer to award sole negotiation rights to the other buyer in exchange for a specified share of the profit. Just below is the "Accept Proposals" box, displaying an offer from the other buyer to give up his rights to buy in exchange for $60 \%$ of the profit; Buyer-B has accepted that offer but can retract by clicking the button. The Chatroom box below contains automatically generated messages from the server as well as messages sent by the traders (none at present). Earnings are shown on the right side of the screen.

profit of the other buyer. ${ }^{8}$ The screens reveal the participant labels, e.g., Buyer A or B, and the seller can view their negotiation in the bulletin board. The upper left area of the collusion screen is where they send proposals and counter proposals negotiating the percentages and who should give up trading. The computer enforces any agreement active at the time of a transaction.

The collusion agreement is retractable unilaterally by either long side trader without penalty prior to a transaction. As soon as either colluding trader clicks a Retract button in market 2, both may resume normal CDA trading, and the retraction is reported in the bulletin board for all to see. Thus the collusion agreement is revocable but is not cheap talk, because DA-Barg enforces the agreement if neither trader had already retracted.

\footnotetext{
${ }^{8}$ DA-Chat allows only a very limited form of profit splitting: if both long siders in a $1 \times 2$ market stick to a pre-play collusion agreement to hold the same price, then they each on average have $50 \%$ chance of being chosen by the buyer for a trade.
} 
Finally, in the structureless bargaining institution (Barg), sellers and buyers can only submit (and adjust or retract) price offers through chat messages, which are visible to all traders in the market. All standing offers are binding and a transaction occurs immediately when a counter-party accepts a standing offer. Because free-form bargaining is more time consuming than posting prices, each period in Barg lasts for 300 seconds.

How are the different trading institutions related to core theory? For both DA-Std and DA-Chat, no transfer is possible between same siders, so they correspond to the restricted game where a trader failing to trade receives zero payoff. As noted in section 3.1, the core prediction still applies. DA-Barg and Barg allow same side transfers, hence they correspond to the full game. Regarding collusion, as discussed in section 3.1, in DA-Std and DA-Chat it simply refers to the failure to undercut a same sider's price; in DA-Barg and Barg, collusion also includes agreeing to same side profit sharing agreements.

\subsection{Procedures}

We recruited 184 subjects from the LEEPS subject pool of over 1000 volunteers, most of them UCSC undergraduates majoring in Economics, Natural Science or Engineering. None had previous experience in this experiment or previous market experiments. On arrival at the laboratory, subjects received printed instructions, reproduced in On-line Appendix C. They then listened to an oral summary with the user interfaces displayed on a wall screen. This was followed by a quiz and a few practice rounds. Over the next 1-2 hours they played 16 to 24 periods of the games described above. Most subjects were paid between $\$ 15$ and $\$ 25$, according to profits earned during the session; average take-home pay was $\$ 22.17$, including a $\$ 5$ show-up fee.

Subjects were randomly rematched each period to avoid repeated game effects. Some sessions had 2 separate marketplaces each with a 2x3 trader configuration each period, and the other sessions had 4 marketplaces each with a 1x2 trader configuration. To maintain anonymity, the messages on screen assigned a label to each participant, e.g. Buyer A or Seller $\mathrm{C}$, and the labels were shuffled every period. The matching scheme guaranteed that the same group of long siders meet at most twice (also, when $n=2$, that short siders meet a minimal number of times) and that they have no way of knowing when they do meet again. 
Consistent with the discussion in section 3.2 above, written and public oral instructions stated the number of participants in each market, e.g., 2 buyers and 3 sellers in a $2 \times 3$ buyers' market, and stated that all buyers had the same value (always 89 lab dollars) and all sellers had the same cost (55 lab dollars). The screen displays immediately made all activities (bids, asks, transactions, proposals, agreements, chat messages, retractions, and so on) public information within the same marketplace. At the end of every period, each subject was shown the transaction prices in all the parallel marketplaces.
DA-Std
DA-Chat
DA-Barg
Barg

\begin{tabular}{lllll}
\hline \hline & & & & \\
1x2: & 2 sessions: & 2 sessions: & 3 sessions: & No sessions: \\
4 markets & B10S10, S10B10 & $\mathrm{B}(2+10) \mathrm{S}(2+10)$, & $\mathrm{B} 10 \mathrm{~S} 10, \mathrm{~B} 10 \mathrm{~S} 10$, due to lesser \\
per period & & $\mathrm{S}(2+10) \mathrm{B}(2+10)$ & $\mathrm{S} 10 \mathrm{~B} 10$ & interest \\
\hline 2x3: & 3 sessions: & 3 sessions: & No sessions: & 4 sessions: \\
2 markets & B8S8, B8S8, S8B8 & $\mathrm{B}(2+6) \mathrm{S}(2+6)$, & due to software & B16, B16, S16, S16 \\
per period & & $\mathrm{B}(2+6) \mathrm{S}(2+6)$, & limitation & \\
& & $\mathrm{S}(2+6) \mathrm{B}(2+6)$ & & \\
\hline \hline
\end{tabular}

Table 1: Experiment Design. Notation such as B8S8 indicates that the session included 8 buyers' market periods followed by 8 sellers' market periods, and $\mathrm{B}(2+10)$ indicates that 2 periods of ordinary CDA preceded 10 augmented periods (with the Chat facility).

Table 1 summarizes the laboratory sessions. In each session, we run either two $2 \times 3$ or four $1 \times 2$ markets simultaneously. The periods in the first half, called segment 1 , of each session are all buyers' markets (or all sellers' markets). Except in the DA-Barg sessions, at the halfway point (that is, after 10 or 12 periods in a $1 \times 2$ market or 8 periods in a $2 \times 3$ market) we switch the role of half (in 1 $\mathrm{x} 2$ sessions) or one third (in $2 \times 3$ sessions) of the long siders, so the switchers stay on the long side throughout the session. These subjects are given double show-up fees; the instructions say that this is "for their role change." Of course, the other subjects who are long (short) siders for segment 1 become short (long) siders for segment 2. A pilot session convinced us that subjects could adapt to the strategic environment more readily with this design - a single switch from buyers' markets to sellers' markets (or the 
reverse) midway through the session - than with alternatives such as simultaneous buyers' and sellers' markets with more rapid rotation of subjects from short side to long side. ${ }^{9}$ Due in part to their slower pace, the Barg sessions employed a between subjects design, either all buyers markets or all sellers market for the entire session.

We ran at least two sessions for each treatment. The relatively high cross-sectional variance encouraged us to run an additional 1x2 DA-Barg session, and the smaller number of markets and lesser number of periods encouraged us to run extra 2x3 sessions.

\subsection{Testable Hypotheses}

Most market experiments analyze transaction price as well as surplus, but in our experiment, surplus split subsumes price since all buyers have the same induced value and all sellers have the same induced cost. We therefore focus on Short Sider Surplus share (SSS) = the fraction of realized surplus obtained by short siders in each transaction.

The experiment allows us to test the following two research hypotheses, plus three supplementary hypotheses concerning our treatment variables.

1. The core prediction is more accurate than the fairness/collusion prediction. Normalizing per-transaction surplus $(89-55=34$ lab dollars $)$ to 1 , the total surplus in an $n \times(n+1)$ market is $n$, so an equal split among the $2 n+1$ players yields $\mathrm{SSS}=n /(2 n+1)$. By contrast, the unique core prediction is $\mathrm{SSS}=1.0$. This first hypothesis is supported when mean SSS exceeds the midpoint $\frac{3 n+1}{4 n+2}$ between equal split and 1.0.

2. The core prediction becomes increasingly accurate over time, and the fairness/collusion prediction eventually becomes less accurate. Under the safe assumption that mean SSS exceeds equal split $n /(2 n+1)$, this hypothesis is supported if there is a positive time trend in SSS.

3. Given the opportunity, subjects will try to collude, but (as predicted by core theory)

\footnotetext{
${ }^{9}$ In the only paid pilot session, we switched buyer/seller assignments three times, seeking to balance profit opportunities across subjects. The final design described in the text seems to allow quicker learning without increasing the perception that the game was unfair.
} 
such attempts will ultimately fail. ${ }^{10}$ This hypothesis is supported if successful collusion (whether or not for an equal split) is rare, especially in later periods.

4. DA-Chat, DA-Barg and Barg were constructed to provide increasing collusion opportunities relative to DA-Std. Hence we expect the core prediction to fare best (i.e., SSS to be largest) in DA-Std and worst (SSS smallest) in Barg.

\section{Results}

To give the reader an overview of the data, we begin with a time graph for a DA-Barg session, mention some idiosyncrasies of other sessions, and then show average SSS for all market institutions. The next subsection presents regressions that test the research hypotheses, and the final subsection explores the supplementary hypotheses. Robustness checks and additional test results are collected in on-line Appendix A.

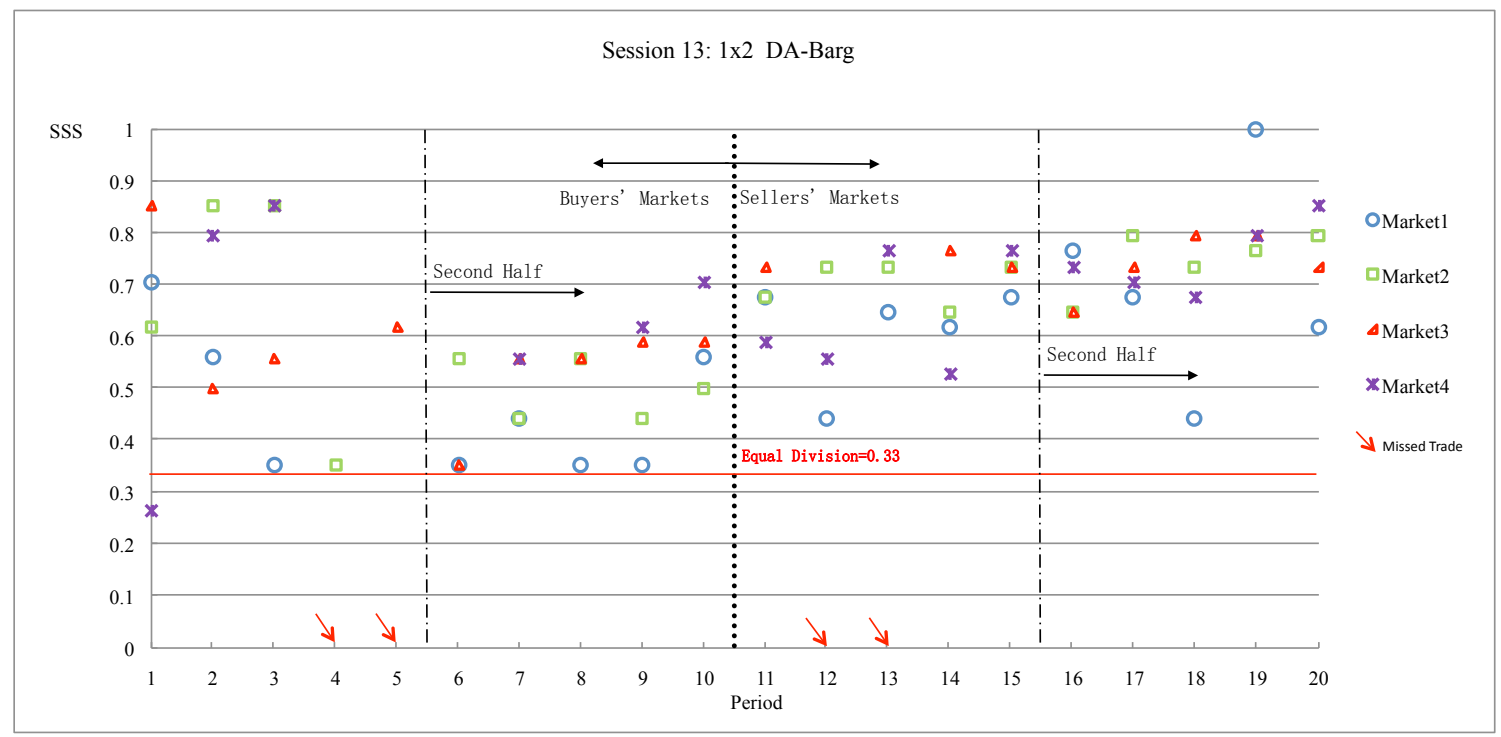

Figure 4: SSS in an Example Session.

\footnotetext{
${ }^{10}$ Some readers might favor the opposite hypothesis (as did one of this paper's coauthors): long siders will become increasingly eager to collude as they come to understand the dire fate that CE holds for them.
} 

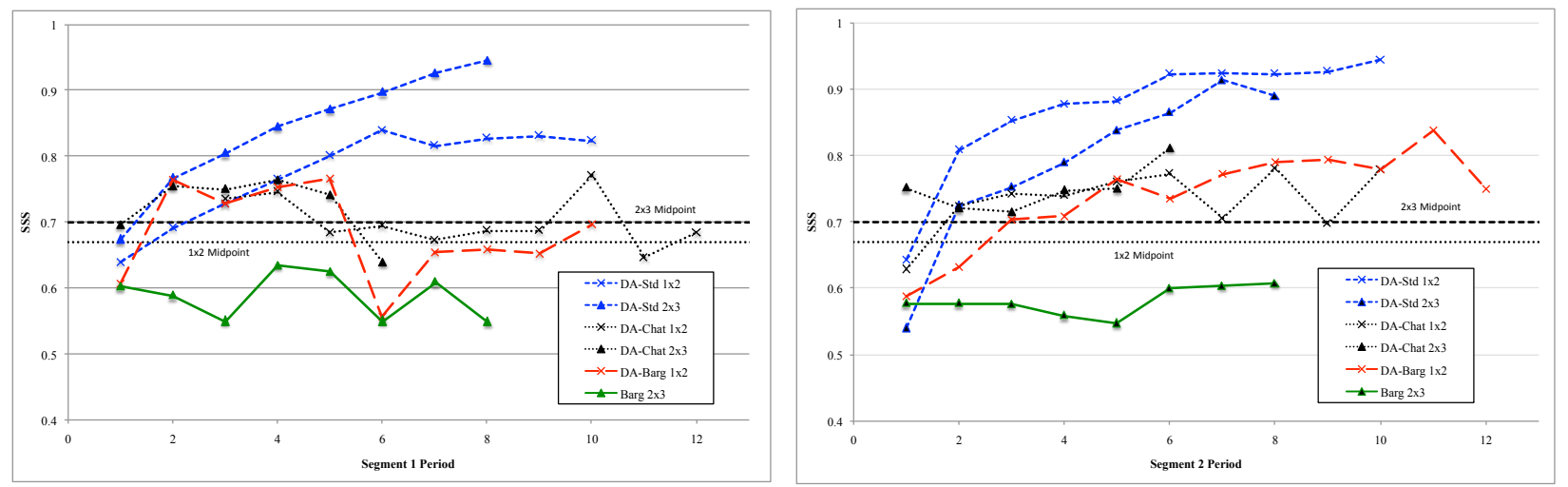

Figure 5: Mean SSS by period in Segment 1 (left panel) and Segment 2 (right panel) for all trading institutions. Between segments, the short side switched from Buyers to Sellers or the reverse.

\subsection{Sample Session and Overview}

Figure 4 summarizes a 1x2 DA-Barg session with 4 short sider buyers and 8 long sider sellers in the first segment of 10 periods; here buyers are short siders ("Buyers' Markets"). In period 11, four sellers became buyers, so the 4 remaining sellers are the short siders (in "Sellers' Markets") in the second segment, comprising periods 11-20. The graph displays the surplus share of each short sider (SSS) each period. Only one trade in the entire session (in period 1) gave a short sider less than $1 / 3$ of the surplus (shown as a horizontal red line labelled "Equal Division"), and the vast majority gave short siders more than $1 / 2$. One sees more approximately .5-.5 splits in this treatment than in most DA-Std and DA-Chat sessions, but there are also many splits that heavily favor the short side. The chatroom transcript suggests that a few short siders offered concessions out of a sense of fairness, ${ }^{11}$ and in general the bargaining style of the individual short sider seems to play an important role. In this session behavior becomes somewhat more consistent in the "Second Half" of the segments (periods 16-20 of Sellers' and periods 6-10 of Buyers' Markets); for example, there were missed trades in periods 4, 5, 12 and 13 but none in the Second Halves. (In many other sessions the First Half is even more erratic. Of course, in about half the sessions the Sellers' Markets segment precedes the Buyers' Markets segment.)

\footnotetext{
${ }^{11}$ E.g., in period 12 , one seller wrote, "because all the sellers are selling at such a high price that sellers are getting a lot more than buyers, which is kinda unfair", and another short sider in period 2 wrote "if we all did that we should all get fair money[.] That is all I am trying to do[,] make it fair."
} 
Figure 5 displays the mean SSS over time for each Segment and each trading institution. ${ }^{12}$

There appear to be substantial differences across trading institutions. The econometric analysis to follow will scrutinize such impressions more carefully, focusing mainly on Second Half data, since (as in many experiments) it includes fewer mistakes and idiosyncrasies. On-line Appendix A includes corresponding econometric analyses of the entire data set; the inferences are often weaker but never contradict those that we now report.

\section{$5.2 \quad$ SSS Regressions}

Hypothesis tests are complicated by three kinds of potential correlation across observations. The two trades in the same $2 \times 3$ market might be correlated, or the trades of a given short sider across periods, or even the trades within the same session. In this subsection we report regressions of SSS that impose random short sider effects and clustering on the sessions level in order to account for potential short sider and session correlation. ${ }^{13}$ On-line Appendix A reports the results for these estimations where the second unit from each 2x3 market is dropped to eliminate the possible within-market correlation; in most cases it makes no appreciable difference, but a couple of minor exceptions (regarding collusion variables) are noted.

Table 2 reports regressions of SSS in each completed Second Half trade on institutional dummies, supplemented by dummies for whether the observations come from the second segment (Seg2 = 1), and whether there are 3 long siders (D2x3 = 1) or just 2. Column 1 of the Table tells us that in DA-Std, the short siders get on average a bit more than $77 \%$ of the surplus in the first (Second Half) period, climbing to over $87 \%(\approx .771+0.060+5 \times 0.009)$ by the last period of the second segment. This far exceeds the midpoint benchmark of $\frac{3 n+1}{4 n+2}=0.70$ in 2x3 sessions and 0.67 in $1 \times 2$ sessions, thus supporting Hypothesis $1 .^{14}$ The positive coefficient

\footnotetext{
${ }^{12}$ The Barg sessions were run later and they had a slightly different format: 16 periods each (except that, due to a software crash, one Barg session ended after 14 periods) with no switch of short siders. For comparative purposes, the Figure treats periods 1-8 as Segment 1 and periods 9-16 as Segment 2.

${ }^{13}$ To be sure that a random effects model is appropriate, we conduct Hausman tests on all specifications in Table 2 and fail to reject that differences in coefficients between a fixed effects and random effects model are not systematic.

${ }^{14}$ Using specification 1 in Table 2 we conduct statistical tests related to Hypothesis 1 . The p-values for the hypothesis that the $1 \times 2$ DA-Std markets are equal to the 0.67 midpoint are 0.029 and 0.000 for the first and last period of the second half, respectively. The p-values for the hypothesis that the 2x3 DA-Std markets are equal to the 0.7 midpoint are 0.001 and 0.000 for the first and last period of the second half, respectively.
} 


\begin{tabular}{lllll} 
Specification & 1 & 2 & 3 & 4 \\
\hline \hline Constant & $0.771^{* * *}$ & $0.855^{* * *}$ & $0.564^{* * *}$ & $0.606^{* * *}$ \\
& $(0.052)$ & $(0.033)$ & $(.025)$ & $(0.026)$ \\
DA-Chat & $-0.163^{* * *}$ & $-0.100^{* * *}$ & & \\
DA-Barg & $(0.037)$ & $(0.036)$ & & \\
Seg2 & $-0.146^{* * *}$ & $-0.085^{*}$ & & \\
D2x3 & $(0.054)$ & $(0.047)$ & & \\
& $0.060^{* *}$ & $0.043^{*}$ & & \\
Trend & $(0.028)$ & $(0.022)$ & & $-0.034^{* *}$ \\
Coll. Prop. & 0.037 & 0.017 & & $(0.017)$ \\
& $(0.037)$ & $(0.031)$ & & $-0.028^{\dagger}$ \\
Coll. Agree & $0.009^{* *}$ & & $0.005^{* * *}$ & $(0.019)$ \\
& $(0.005)$ & & & $-0.026^{\dagger}$ \\
Hon. Agree & & $-0.062^{* * *}$ & & $(0.016)$ \\
Fairness & & $(0.021)$ & & $-0.033^{\dagger}$ \\
Data Range & & $-0.076^{* * *}$ & & $(0.021)$ \\
R-sq (overall) & 0.295 & $(0.012)$ & & Barg Sessions \\
Rho & & $-0.180^{* * *}$ & & 0.030 \\
Observations & 422 & $(0.033)$ & & 0.373 \\
\hline
\end{tabular}

Table 2: SSS regression coefficient estimates (and standard errors). The treatment dummies (DAChat, DA-Barg, D2x3 and Seg2) are defined in the text. Trend is the number of periods elapsed from the beginning of each segment. The collusion variables (Coll. Prop., ..., Fairness) are defined later in the text. Two-sided significance at $1 \%, 5 \%$ and $10 \%$ levels are indicated respectively by ${ }^{* * *},{ }^{* *}$ and *, while ${ }^{\dagger}$ indicates one-sided significance at the $10 \%$ level. Rho indicates the fraction of variation explained by individual short sider random effects. Standard errors are clustered at the session level.

for D2x3 suggests if anything stronger support for that core prediction when the market is larger. The significant coefficients for DA-Chat and DA-Barg (ranging from -0.085 to 0.163 depending on the specification) indicate the evidence is less extreme but, as we will see in subsection 5.4, it still is favorable to this research hypothesis for these other market institutions.

On the other hand, Column 3 of the Table does not support Hypothesis 1 for the unstructured Barg institution: the estimated SSS is $0.564+16 \times 0.005=0.644<0.70$ in the last 
period of a Barg segment. The significantly positive trend coefficients in both columns support Hypothesis 2, that SSS increases over time. Supplementary Hypothesis 4 is supported in that DA-Std yields the largest SSS and Barg the smallest; the other two DA institutions are in between and statistically indistinguishable from each other. ${ }^{15}$ Columns 2 and 4 are relevant to supplementary Hypothesis 3 regarding collusion, as we now shall see.

\subsection{Collusion}

Two research assistants independently examined the chatroom transcripts and collusion market logs for all DA-Chat, DA-Barg and Barg sessions. They determined, for each market of each period, whether collusion was proposed (Coll. Prop. in Table 2), whether fairness was discussed (Fairness), whether there was agreement on a proposal (Coll. Agree), and whether offers conformed to an agreement (Hon. Agree). Collusion was deemed successful if offers conforming to an agreement resulted in a transaction. No collusion attempt was found on the short side in the DA treatments; of the 16 attempts observed in Barg, 14 occurred in the first half of the session. We conclude that short side collusion in our experiment is an artifact of subject inexperience, and so the results below pertain to long side collusion only. Online Appendix B includes more details and reports the evidence (quite minimal) on tacit collusion.

Columns 2 and 4 of Table 2 show that all degrees of collusion are significantly associated with lower SSS. Column 2 indicates that, absent collusive agreements, about $90 \%(\approx 0.855+$ 0.043 in 1x2 sessions) of SSS in segment 2 goes to the short side in DA-Std and only 9 or 10\% less in DA-Chat and DA-Barg sessions. However, even proposing collusion is associated with about a $6 \%$ reduction in SSS, agreed collusion with a $7.6 \%$ reduction, and honored collusion agreements with a $18 \%$ reduction in SSS. Fairness discussion alone is much less significant here, economically and statistically. Column 4 shows a much lower baseline SSS in Barg, further reduced by about $3 \%$ given any sort of collusion.

\footnotetext{
${ }^{15}$ The coefficients on DA-Chat and DA-Barg in specification 1 are both negative and statistically significant at the $1 \%$ level, which is evidence that the SSS in DA-Std is statistically larger than the other two DA treatments. Using results from specification 1 a pairwise test on the difference between DA-Chat and DABarg fails to reject that their difference is equal to zero (p-value of 0.72). A supplementary regression for specification 1 was conducted that combined the DA and Barg institutions. A pairwise test on the difference between DA-Chat (the lowest DA SSS) and Barg sessions rejects that their difference is equal to zero (p-value of 0.0323) and thus provides statistical support that Barg sessions produce the lowest SSS.
} 


\begin{tabular}{|c|c|c|c|c|c|}
\hline Specification & 1 & $1 \mathrm{a}$ & 2 & 3 & 4 \\
\hline Dep. Var. & $\begin{array}{l}\text { Successful } \\
\text { Collusion }\end{array}$ & $\begin{array}{l}\text { Successful } \\
\text { Collusion }\end{array}$ & Missed Trade & $\begin{array}{l}\text { Successful } \\
\text { Collusion }\end{array}$ & "Missed Trade \\
\hline Constant & $\begin{array}{l}-1.816^{* * *} \\
(0.513)\end{array}$ & $\begin{array}{l}-3.420^{* * *} \\
(0.725)\end{array}$ & $\begin{array}{l}-3.893^{* * *} \\
(0.746)\end{array}$ & $\begin{array}{l}-2.981^{* * *} \\
(0.493)\end{array}$ & $\begin{array}{l}-2.008^{* * *} \\
(0.386)\end{array}$ \\
\hline DA-Chat & & & $\begin{array}{l}1.523^{* *} \\
(0.720)\end{array}$ & & \\
\hline DA-Barg & $\begin{array}{l}0.803^{\dagger} \\
(0.555)\end{array}$ & $\begin{array}{l}1.603^{* *} \\
(0.648)\end{array}$ & $\begin{array}{l}1.409^{*} \\
(0.807)\end{array}$ & & \\
\hline Seg2 & $\begin{array}{l}-0.726^{\dagger} \\
(0.525)\end{array}$ & $\begin{array}{l}-0.843^{\dagger} \\
(0.587)\end{array}$ & $\begin{array}{l}-0.097 \\
(0.439)\end{array}$ & & \\
\hline Smarket & $\begin{array}{l}-1.851^{* * *} \\
(0.651)\end{array}$ & $\begin{array}{l}-1.448^{* *} \\
(0.696)\end{array}$ & $\begin{array}{l}0.079 \\
(0.347)\end{array}$ & $\begin{array}{l}0.519^{\dagger} \\
(0.390)\end{array}$ & $\begin{array}{l}0.024 \\
(0.362)\end{array}$ \\
\hline $\mathrm{D} 2 \times 3$ & $\begin{array}{l}-0.938 \\
(1.130)\end{array}$ & $\begin{array}{l}-1.203 \\
(1.176)\end{array}$ & $\begin{array}{l}-0.294 \\
(0.635)\end{array}$ & & \\
\hline Fairness & & $\begin{array}{l}2.488^{* * *} \\
(0.595)\end{array}$ & & $\begin{array}{l}1.714^{* * *} \\
(0.472)\end{array}$ & $\begin{array}{l}1.275^{* * *} \\
(0.398)\end{array}$ \\
\hline Second Half & & & & $\begin{array}{l}-0.591^{\dagger} \\
(0.390)\end{array}$ & $\begin{array}{l}-1.132^{* * *} \\
(0.388)\end{array}$ \\
\hline Data Range & $\begin{array}{l}2 \mathrm{H} \text { of DA- } \\
\text { Barg, -Chat }\end{array}$ & $\begin{array}{l}2 \mathrm{H} \text { of DA- } \\
\text { Barg, -Chat }\end{array}$ & $2 \mathrm{H}$ of All DA & $\begin{array}{l}\text { All Barg Ses- } \\
\text { sions }\end{array}$ & $\begin{array}{l}\text { All Barg Ses- } \\
\text { sions }\end{array}$ \\
\hline Log likelihood & -60.14 & -50.07 & -85.95 & -90.43 & -99.78 \\
\hline Observations & 231 & 231 & 445 & 252 & 252 \\
\hline
\end{tabular}

Table 3: Logit regressions with random session effects (and standard errors). Data from all periods are included. Explanatory variables and significance flags are as defined in the previous table, e.g., ${ }^{\dagger}$ indicates significance at the one-sided $10 \%$ level. The label All as opposed to $2 \mathrm{H}$ indicates that first half data are included as well as second half data.

Table 3 reports logit regressions that further dissect the collusion process. It includes random session effects to control for the fact that long siders encourage one another to collude and are rematched every period. ${ }^{16}$ Column 1 shows that there are significantly more successful collusions in DA-Barg than in DA-Chat (the omitted dummy in this column), and in buyers markets than in sellers markets. Successful collusion is more prevalent in 1x2 sessions than in 2x3 sessions. Consistent with Hypothesis 3, collusion is less prevalent in segment 2 than in segment 1: although only marginally statistically significant, the coefficient estimate is economically significant — e.g., the estimated intercept and Seg2 coefficients of -1.816 and

\footnotetext{
${ }^{16}$ E.g., one long sider in session 12 advocated collusion to every fellow long sider he met and another long sider in the same session even asked other long siders to "spread the word."
} 
-0.726 imply that the rate of successful collusion drops from about $14 \%$ in the first segment to about $7 \%$ in the second segment in a 1x2 DA-Chat buyer's market. The fitted logit equation implies (and examination of raw data confirm) similarly impressive drops in successful collusion for all institutions except, of course, DA-Std. Column 1a shows that appeals to fairness are very significantly associated with successful collusion. The loss of marginal significance for Seg2 seems attributable to a declining number of fairness appeals.

Column 3 confirms some of these tendencies in the Barg sessions. (Here, since we don't switch roles, we include first half as well as second half data.) Fairness again is strongly and positively associated with successful collusion and, consistent with Hypothesis 3, successful collusion is again marginally significantly less prevalent in the second half of these single segment sessions. Since we have no a priori hypothesis about whether seller or buyer markets are more conducive to successful collusion, a single tailed test is inappropriate and the positive estimated coefficient should not be considered significant at even the $10 \%$ level. We have no explanation for the significantly negative coefficients on this variable in columns 1 and 1a, but note that they seem consistent with the results of Joyce (1984) mentioned earlier.

Columns 2 and 4 of the table show that inefficiency, which takes the form of missed trades, moves in rough parallel with successful collusion. The estimates imply efficiencies in 1x2 buyer's market second segments between 92.2\% DA-Barg and 98.2\% DA-Std, with perhaps slightly higher efficiencies in 2x3 markets. The implied efficiency in Barg is $88.2 \%$.

\subsection{Final Surplus Split}

We can combine our two research hypotheses to come to an overall conclusion about the predictive power of the core. Table 4 uses coefficient estimates to compute (or extrapolate) final SSS values, where for the sake of consistency we define the final period to be 8 periods from the the beginning of the second half of (where applicable) segment 2. The coefficient estimates come from a regression, reported in Table A3 of the Appendix, that includes interaction terms to account for the possibility that time trends differ across treatments and across segments. These estimated final SSS values come with standard errors that imply the given 95\% confidence intervals and the indicated significance levels for rejecting the null hypothesis that the estimated values are equal to the $\frac{3 n+1}{4 n+2}$ benchmark. As a consistency check, the table 
also reports the mean SSS directly observed in the last two periods for each treatment cell.

\begin{tabular}{lcccc} 
& DA-Std & DA-Chat & DA-Barg & Barg \\
\hline \hline $1 \times 2$ & $0.922^{* * *} \pm 0.033$ & $0.746^{* * *} \pm 0.029$ & $0.836^{* *} \pm 0.037$ & \\
(midpoint=0.67) & {$[0.856-0.987]$} & {$[0.690-0.803]$} & {$[0.763-0.909]$} & \\
Last 2 Actual & $0.843^{* * *} \pm 0.026$ & $0.741^{* *} \pm 0.034$ & $0.824^{* * *} \pm 0.030$ & \\
& & & & \\
$2 \times 3$ & $0.960^{* * *} \pm 0.036$ & $0.773 \pm 0.057$ & & $0.600 \pm 0.0344$ \\
(midpoint=0.70) & {$[0.890-1.03]$} & {$[0.661-0.883]$} & & {$[0.535-0.666]$} \\
Last 2 Actual & $0.881^{* * *} \pm 0.019$ & $0.778^{* *} \pm 0.035$ & & $0.606 \pm 0.021$ \\
\hline
\end{tabular}

Table 4: End of session fitted SSS values \pm standard errors. The fitted values are generated using specification in Table A3 in the Appendix. Here ${ }^{* * *},{ }^{* *}$ and ${ }^{*}$ indicate two-sided significance at $1 \%, 5 \%$ and $10 \%$ levels respectively (and ${ }^{\dagger}$ indicates $10 \%$ one-sided significance) in rejecting the null hypothesis that the fitted value is equal to the midpoint between the core prediction and the egalitarian prediction (in favor of the alternative that it is closer to the core prediction). Centered 95\% confidence intervals are shown [in brackets]. Last 2 Actual reports the mean \pm standard deviation of SSS observed in the last two periods of all markets in each cell.

Table 4 shows that the core prediction is far better than the egalitarian prediction for DAStd for both $1 \times 2$ and 2x3 markets and for DA-Barg; the evidence is significant and favorable for $1 \times 2$ DA-Chat as well. The evidence is less significant but still favorable for 2x3 DA-Chat. However, the egalitarian prediction is better than the core prediction for Barg.

\subsection{Summary}

Our laboratory experiment explores the predictive power of the core for several different trading institutions. Our first set of sessions considered a standard continuous double auction (DA-Std), which is known to be capable of delivering very asymmetric payoffs, and two augmentations that enabled free-form pre-play chat (DA-Chat) and that also enforced agreements (DA-Barg). For all institutions in this first set, the evidence from both 1x2 markets and 2x3 markets favored (and for DA-Std, strongly favored) the extreme core prediction that the short siders reap the entire surplus over the alternative egalitarian prediction that short siders would on average do no better than long siders. Moreover, consistent with convergence towards the core prediction, we detect a downward trend in collusion (successful and otherwise) in both augmented institutions. 
Follow-up sessions investigated a version of free-form bargaining, which is generally known to favor equal splits of available surplus; classic textbook discussions include Davis and Holt (1993)(p. 243) and Camerer (2003)(pp. 153-161). Here the predictive power of the core is less impressive. Although the time trends in SSS (and collusion) continue to favor the core, the egalitarian prediction of surplus split is more accurate than the core prediction even in the last period.

Hypothesis 1 (that core predictions are more accurate than equal split) thus is supported for the standard DA and both augmentations, but not for the free-form bargaining institution. Hypothesis 2 (that the time trend favors the core prediction) is supported for all institutions. Supplementary Hypothesis 3 (that sucessful collusion is infrequent and trends downward) is also supported, as is supplementary Hypothesis 4, that core predictions work best for DA-Std and worst for Barg.

We were a bit surprised that the data do not support a stronger version of Hypothesis 4, the conjecture that short siders would reap a larger fraction of surplus in DA-Chat than in DA-Barg. The analysis presented above leaves the ranking unclear between these two augmented DA institutions. Finer grained regressions reported in Appendix A suggest that the conjecture may be true in earlier periods but false in later periods.

\section{Discussion}

What do our results tell us about the longstanding critique of the core? In our lab experiment, the extreme sensitivity to one extra player can be quite realistic after all. Indeed the example is a credit to the core as it successfully predicts a counterintuitive outcome for some reasonable trading institutions. Due to the inherent connection between the core and the competitive equilibrium, our results also contribute to the literature on the boundaries of CE. In particular they demonstrate robustness of CE in very thin markets with extreme supply-demand configurations and unusual collusion opportunities.

We also find that the core prediction has its limits. In follow-up sessions with a free-form bargaining institution, we obtained outcomes slightly closer to the egalitarian prediction of equal gains for everyone than to the extreme core prediction of zero gains for the side with 
more traders.

We hope that our results provoke further thought and analysis on the roles of fairness, collusion and strategic advantage. We adopted a priori a rather simplistic notion of fairness as equal split of surplus among all market participants in any given market period, but we recognize that fairness is a notoriously slippery concept with many interpretations. Two are worth mentioning here. ${ }^{17}$ First, as noted by an anonymous referee, human subjects may consider fairness in terms of earnings over an entire laboratory session rather than one period at a time, and if so the core prediction might appear better than it would in a true one-shot setting. Chat transcripts from a pilot session that involved multiple role switches suggested that possibility to us as well, but we see very little evidence of such thinking in our reported sessions, which have at most a single, unadvertised switch of a few subjects between buyer and seller roles.

Second, human subjects might take a narrower, transaction-by-transaction view of fairness. It might be considered fair for the short sider in every transaction to take half the surplus and the lucky long sider to take the other half, leaving nothing for the unlucky. With that interpretation, the midpoint between the core prediction and the fair (but not egalitarian) split of surplus would always be SSS $=0.75$. The conclusions regarding Hypothesis 1 would be unchanged for DA-Std, DA-Barg and Barg, but the confidence intervals for the final surplus split in DA-Chat would now include that midpoint.

Collusion seems to decline over time in all our treatments. In partial contrast, the short siders' inherent strategic advantage seems to gain strength over time in most of our institutions, but perhaps not in DA-Chat, the institution that allows only cheap-talk collusion. Another possible hint (also gleaned from Appendix A) is that subjects typically do not wait until the last moment to agree on a surplus split; on average they transact halfway through the trading period. As noted in the text, appeals to fairness are strongly associated with successful collusion (and with missed trades).

We hope theorists find these hints helpful in constructing noncooperative models of trade in settings such as ours. One possible direction is to model a trader's reservation price at terms worse than which she would rather miss the trade - as depending on fairness

\footnotetext{
${ }^{17}$ Readers interested in learning more about formal economic models of fairness may wish to read the survey of Cooper and Kagel (2009).
} 
considerations. Bargaining using such reservation prices might do better than tacit collusion or other approaches in capturing the empirical regularities just mentioned.

Our study also opens the way to future experimental work. For example one could examine other market institutions such as the call market, in which bids and asks are submitted simultaneously and then a market-clearing price is generated to determine all the transactions. Generally the call market converges less quickly and is somewhat less efficient than DA-Std, but it is not clear how it would fare in our environment. Another possibility for further study is to try reducing or eliminating the sense of unfairness in the core allocation, for example by taxing short siders' profits. Finally, it surely would be interesting to extend the so-far quite limited experimental literature on noncooperative foundations of the core.

\section{References}

Camerer, C., 2003. Behavioral game theory: Experiments in strategic interaction. Princeton University Press.

Cason, T. N., Noussair, C., 2007. A market with frictions in the matching process: An experimental study. International Economic Review 48 (2), 665-691.

Cohen, J., et al., 1960. A coefficient of agreement for nominal scales. Educational and psychological measurement $20(1), 37-46$.

Cooper, D., Kagel, J. H., 2009. Other regarding preferences: a selective survey of experimental results. Handbook of experimental economics 2.

Davis, D. D., Holt, C. A., 1993. Experimental economics. Princeton university press.

Dufwenberg, M., Gneezy, U., 2000. Price competition and market concentration: an experimental study. International Journal of Industrial Organization 18 (1), 7-22.

Eavey, C. L., Miller, G. J., 1984. Fairness in majority rule games with a core. American Journal of Political Science, 570-586. 
Edgeworth, F. Y., 1881. Mathematical psychics: An essay on the application of mathematics to the moral sciences. No. 10. CK Paul.

Fiorina, M. P., Plott, C. R., 1978. Committee decisions under majority rule: An experimental study. American Political Science Review 72 (02), 575-598.

Friedman, D., Ostroy, J., 1995. Competitivity in auction markets: An experimental and theoretical investigation. The Economic Journal, 22-53.

Gillies, D. B., 1954. Some theorems on n-person games. Ph.D. thesis, Princeton University.

Harrison, G. W., McKee, M., 1985. Monopoly behavior, decentralized regulation, and contestable markets: An experimental evaluation. The RAND Journal of Economics, 51-69.

Huck, S., Normann, H.-T., Oechssler, J., 2004. Two are few and four are many: number effects in experimental oligopolies. Journal of Economic Behavior \& Organization 53 (4), 435-446.

Isaac, R. M., Ramey, V., Williams, A. W., 1984. The effects of market organization on conspiracies in restraint of trade. Journal of Economic Behavior \& Organization 5 (2), 191-222.

Joyce, P., 1984. The walrasian tatonnement mechanism and information. The RAND Journal of Economics, 416-425.

Murnighan, J. K., Roth, A. E., 1977. The effects of communication and information availability in an experimental study of a three-person game. Management Science 23 (12), 1336-1348.

Murnighan, J. K., Roth, A. E., 1978. Large group bargaining in a characteristic function game. Journal of Conflict Resolution 22 (2), 299-317.

Murnighan, J. K., Roth, A. E., 1980. Effects of group size and communication availability on coalition bargaining in a veto game. Journal of Personality and Social Psychology 39 (1), 92.

Perry, M., Reny, P. J., 1994. A noncooperative view of coalition formation and the core. Econometrica: Journal of the Econometric Society, 795-817. 
Roth, A. E., Prasnikar, V., Okuno-Fujiwara, M., Zamir, S., 1991. Bargaining and market behavior in jerusalem, ljubljana, pittsburgh, and tokyo: An experimental study. The American Economic Review, 1068-1095.

Selten, R., 1980. A noncooperative model of characteristic-function bargaining. Institut für Mathematische Wirtschaftsforschung an der Universität Bielefeld.

Shapley, L. S., 1952. Notes on the n-person game, III: Some variants of the von NeumannMorgenstern definition of solution. Rand Corporation.

Smith, V. L., Williams, A. W., 1990. The boundaries of competitive price theory: Convergence, expectations, and transaction costs. Advances in behavioral economics 2, 3-35.

Yan, H., 2003. Noncooperative selection of the core. International Journal of Game Theory $31(4), 527-540$. 